\title{
Incidência de lesão por pressão em pacientes na unidade de terapia intensiva de um hospital filantrópico
}

RESUMO | O objetivo desta pesquisa foi determinar a incidência e analisar o perfil dos portadores de lesão por pressão, enfocando fatores de risco, características clínicas e demográficas dos pacientes internados em Unidade de Terapia Intensiva (UTI) do hospital IMIP, assim como estádio e localização das lesões no corpo. Trata-se de um estudo descritivo, documental, com abordagem quantitativa. Realizado na UTI Cirúrgica do complexo hospitalar do Instituto de Medicina Integral Professor Fernando Figueira (IMIP), localizado em Recife no estado de Pernambuco, através da coleta de dados dos censos diários e do uso da escala de Braden com um formulário específico durante o período de outubro de 2018 a dezembro de 2018. Percebe-se a importância de um trabalho em equipe, com planejamento estratégico, buscando sempre solucionar as falhas não de forma isolada, mas em conjunto, seja na área médica, da enfermagem, nutricional, fisioterapia, e dos outros profissionais envolvimentos no cuidado do paciente internado nesta unidade.

Palavras-chaves: Lesão por Pressão; Indicadores de Qualidade em Assistência à Saúde; Unidade de Terapia Intensiva.

ABSTRACT | The aim of this research was to determine the incidence and analyze the profile of patients with pressure injuries, focusing on risk factors, clinical and demographic characteristics of patients admitted to the Intensive Care Unit (ICU) of the IMIP hospital, as well as the stage and location of the injuries on body. This is a descriptive, documentary study, with a quantitative approach. Held in the Surgical ICU of the hospital complex of the Instituto de Medicina Integral Professor Fernando Figueira (IMIP), located in Recife in the state of Pernambuco, through the collection of data from the daily censuses and the use of the Braden scale with a specific form during the period of October 2018 to December 2018. One realizes the importance of teamwork, with strategic planning, always seeking to resolve failures not in isolation, but together, whether in the medical, nursing, nutritional, physical therapy, and of other professionals involved in the care of the patient admitted to this unit.

Keywords: Pressure Injury; Health Care Quality Indicators; Intensive Care Unit.

RESUMEN | El objetivo de esta investigación fue determinar la incidencia y analizar el perfil de los pacientes con lesiones por presión, enfocándose en los factores de riesgo, las características clínicas y demográficas de los pacientes ingresados en la Unidad de Cuidados Intensivos (UCI) del hospital IMIP, así como el estadio y la ubicación de las lesiones. en el cuerpo. Este es un estudio documental descriptivo, con un enfoque cuantitativo. Realizado en la UCI Quirúrgica del complejo hospitalario del Instituto de Medicina Profesor Integral Fernando Figueira (IMIP), ubicado en Recife en el estado de Pernambuco, a través de la recopilación de datos de los censos diarios y el uso de la escala de Braden con una forma específica durante el período de Octubre de 2018 a diciembre de 2018. Uno se da cuenta de la importancia del trabajo en equipo, con planificación estratégica, siempre buscando resolver las fallas no de forma aislada, sino en conjunto, ya sea en la medicina, enfermería, nutrición, fisioterapia y de otros profesionales involucrados en el cuidado del paciente ingresado en esta unidad.

Palavras claves: Lesión por Presión; Indicadores de Calidad de Atención Médica; Unidad de Terapia Intensiva.

\section{Jonata Bruno da Silva Santos}

Graduando em Enfermagem pela Uninassau. Hospital Unimed, Otávio de Freitas e Getúlio Vargas. Recife, PE/Brasil.

\section{Marcos Antonio de Oliveira Souza}

Enfermeiro. Doutor em Promoção à Saúde. Professor da Uninassau e enfermeiro do Hospital Getúlio Vargas. Recife, PE/Brasil.

\section{Ana Paula Arruda da Silva}

Enfermeira. Fundação de Ensino Superior de Olinda

Milena Bianca da Silva

Enfermeira. Faculdade pernambucana de saúde

\section{Vitória Marion Costa Silva}

Enfermeira. Faculdade pernambucana de saúde

Roberta Moraes Nogueira

Fisioterapeuta. Faculdade pernambucana de saúde

Recebido em: 11/05/2020

Aprovado em: 13/05/2020
INTRODUÇÃO

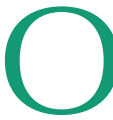
Ministério da Saúde, através da Portaria n. 466 de 1998, define Unidade de Terapia Intensiva (UTI) como um setor que "[...] constitui-se de um conjunto de elementos funcionalmente agrupados, destinado ao atendimento de pacientes graves ou de risco, que exijam assistência médica e de enfermagem ininterruptas, além de equipamentos e recursos humanos especializados $^{\prime \prime(1)}$.

O papel da UTI é de suma importância no aumento da sobrevida de pacientes criticamente enfermos, desde aqueles normalmente vítimas de traumas, disfunções neurológicas, respiratórias ou de qualquer outro tipo. Ambiente este composto por equipe multidisciplinar, onde se presta assistência qualificada, especializada e observação constante ${ }^{(2)}$.

Nesse contexto, fatores que alteram nossa realidade social e epidemiológica, como a introdução de tecnologias avançadas, o aumento do número de idosos, novas doenças e o aumento do índice de adoecimento por doenças crônicas que necessitam de cuidados intensivos, em algum momento, têm gerado uma nova demanda assistencial, determinando a rápida evolução de novas especialida$\operatorname{des}^{(3)}$. Por conseguinte, as UTIs têm sido uma estratégia para o oferecimento de um suporte especializado de assistência à saúde, sendo um dos setores mais complexos dentro do ambiente hospitalar, e exige o uso de recursos tecnológicos e terapêuticos de ponta. Dessa forma, esses são alguns fatores que contribuem para 
a elevação dos custos relacionados aos cuidados intensivos, sendo de suma importância que o tratamento dos pacientes internados neste setor tenha uma boa relação custo-eficiência ${ }^{(4)}$.

Muito embora a UTI aumente a sobrevida do paciente, os efeitos deletérios da imobilidade e exposição a ela merecem atenção. Devido à condição clínica do paciente e à variedade de procedimentos invasivos realizados, o risco de adquirir uma infecção aumenta drasticamente ${ }^{(5)}$. Por outro lado, a imobilidade contribui significativamente para o declínio funcional do paciente, aumentando a incidência de complicações neuromusculares, pulmonares, cognitiva, lesões por pressão e piora na qualidade de vida ${ }^{(6)}$.

A National Pressure Ulcer Advisory Panel (NPUAP) padronizou em 2016 um novo conceito sobre úlcera por pressão (lesão por pressão) e seu estadiamento. Atualmente, a úlcera por pressão é denominada "lesão por pressão" e é definida como:

\begin{abstract}
"dano localizado na pele e/ ou tecidos moles subjacentes, geralmente sobre uma proeminência óssea ou relacionada ao uso de dispositivo médico ou a outro artefato. A lesão pode se apresentar em pele íntegra ou como úlcera aberta, e pode ser dolorosa. A lesão ocorre como resultado da pressão intensa e/ou prolongada em combinação com o cisalhamento. A tolerância do tecido mole à pressão e ao cisalhamento pode também ser afetada pelo microclima, nutrição, perfusão, comorbidades e pela sua condição"(7).
\end{abstract}

Neste contexto, a Organização Mundial de Saúde (OMS) utiliza a incidência e a prevalência das lesões por pressão como um dos indicadores para determinar a qualidade dos cuidados prestados, pois cerca de 95\% das LPs são evitáveis, pelo que se torna imprescindível utilizar todos os meios disponíveis para realizar uma eficaz prevenção e tratamento das LPs já estabelecidas ${ }^{(8)}$. Estudo realizado em 22 UTIs, de 15 hospitais de Belo Horizonte, identificou que a ocorrência de, pelo menos, uma lesão por pressão por paciente foi de $35,2 \%$. Na literatura internacional, há uma prevalência que varia de 4,0 a $49,0 \%$ e uma incidência de 3,8 a $12,4 \%$ em ambiente de cuidados intensivos ${ }^{(9)}$.

No Brasil, estudos têm avaliado a incidência e a prevalência de LP tanto no ambiente hospitalar quanto nas instituições de longa permanência e nos domicílios, revelando que os números variam de acordo com o cenário e o perfil dos pacientes estudados, situando-se a incidência entre $3,6 \%$ e $66,6 \%{ }^{(10)}$.

Nessa circunstância, a prevenção acaba sendo o principal foco dos profissionais responsáveis pelo cuidado do paciente. A abordagem preventiva deve ser multidisciplinar e tem início na identificação precoce dos pacientes suscetíveis, devendo abranger a equipe cuidadora, além dos familiares envolvidos e do próprio paciente, quando possível. Mecanismos de distribuição da pressão, mudança periódica de posição, controle da incontinência, cuidados com a pele e nutrição são as principais medidas envolvidas ${ }^{(11)}$.

Um dos mecanismos de prevenção é a Escala de Braden, que foi desenvolvida com base na fisiopatologia das LPs, utilizando três determinantes: a intensidade, a duração da pressão e a tolerância tecidual. Ela apresenta seis subescalas: percepção sensorial, umidade da pele, atividade, mobilidade, estado nutricional e fricção e cisalhamento, que são pontuados de um a quatro, com exceção da fricção e do cisalhamento, cuja pontuação varia de um a três. Os escores totais variam de seis a 23, onde os valores mais altos indicam um baixo risco de formação de LPs, e os baixos escores indicam um alto risco para a ocorrência dessas lesões ${ }^{(12)}$.

Este estudo tem por objetivo identificar a incidência de LP em pacientes 
internados nas UTIs em um complexo hospitalar relacionando com suas principais causas.

\section{METODOLOGIA}

Trata-se de um estudo transversal, documental e analítico de natureza quantitativa, cujo local de estudo deu-se no âmbito na UTI adulto do Instituto de Medicina Integral Professor Fernando Figueira (IMIP), localizado no Bairro da Boa Vista, na cidade do Recife, Pernambuco. Esta UTI apresenta 10 leitos, admitindo pacientes provindos de cirurgias de pequeno, médio e grande porte nas seguintes especialidades: vascular, otorrinolaringologia, cirurgia geral, ortopedia, plástica, cabeça e pescoço, ginecologia e urologia, ocasionalmente admitindo também pacientes transplantados e do Serviço de Pronto Atendimento (SPA). A população foi composta de pacientes internados na UTI adulto do complexo hospitalar IMIP, que apresentaram riscos de desenvolvimento de lesões por pressão de acordo com a escala de Braden, que consta com um total de 91 pacientes de ambos os gêneros. O período de coleta foi de outubro a dezembro de 2018.

Foi uma amostra sequencial composta por todos os prontuários de pacientes da UTI adulto do complexo hospitalar IMIP que estiveram completos para recoIhimento de dados e que foram admitidos durante o período do estudo. Como se tratou de um estudo de série de casos, não foi necessário cálculo amostral.

Para o início da coleta de dados, primeiramente foi apresentado o projeto à Coordenadora do setor da pesquisa e à Diretoria do centro de saúde, a fim de viabilizar o projeto através da autorização para realizar a pesquisa cedida de forma documental através da carta de anuência. Assim, o projeto foi submetido ao Comitê de Ética em Pesquisa (CEP) com seres humanos da instituição. Após parecer favorável do CEP, iniciou-se a coleta, cujos critérios de inclusão foram pacientes com prontuários completos e que aceitassem participar da pesquisa através da assinatura do Termo de Consentimento Livre e Esclarecido (TCLE), pacientes avaliados com escala de Braden com risco de desenvolver LP e pacientes com idade entre 18 a 80 anos. E como critérios de exclusão, pacientes que, mesmo após a assinatura do TCLE, manifestassem interesse de não participar mais da pesquisa ou pacientes já admitidos com LP.

Durante o período do estudo foram realizadas visitas diárias na UTI do complexo hospitalar IMIP em busca dos prontuários para coleta dos dados pessoais e de identificação, dados de internação, diagnóstico, comorbidades associadas, origem do paciente, gravidade clínica, indicadores de mortalidade e funcionalidade. Todos os dados foram coletados através de uma ficha de coleta de dados, construída para esse estudo e após manifestação de interesse em participar da pesquisa por meio de TCLE. Os dados foram registrados em planilha no Microsoft Excel ${ }^{\circledR}$ com dupla entrada para validação dos dados, eliminando possíveis erros de digitação. Após a validação os dados, foram transportados para o programa Epilnfo® versão 7 para aplicação da análise estatística. Os resultados serão apresentados em gráficos de frequência.

A pesquisa é regida pela Resolução n. ${ }^{\circ}$ 466/2012 do Conselho Nacional de Saúde (CNS) e conta com a carta de anuência e com o Parecer n. ${ }^{\circ}$ 2.836.607 do CEP da Faculdade Pernambucana de Saúde da cidade do Recife - PE. E possui riscos mínimos de exposição de dados do paciente, que foram amenizadas pela garantia do sigilo da pesquisa. Como benefícios, a pesquisa serve como instrumento para avaliar e reconhecer os fatores que favorecem o desenvolvimento das lesões por pressão e propor medidas para minimizar essa ocorrência, fornecendo subsídios para criação de métodos gerenciais que possam diminuir a incidência em ambiente de UTI, promovendo uma meIhor qualidade assistencial.

Os dados ficarão sob a guarda dos pesquisadores por cinco anos, sendo garantido seu sigilo e confidencialidade.

\section{RESULTADOS}

No período, 90 pacientes participaram da pesquisa após assinatura do TCLE, todos apresentavam algum risco de desenvolvimento de lesão por pressão, segundo escala de Braden. Foram identificadas 05 notificações de LP neste período, correspondendo a uma incidência de 5,6\% do total da amostra, conforme Gráfico 1.
Gráfico 1. Incidência de Lesão por Pressão. Recife, PE, Brasil, 2018

\section{Incidência de Lesão por Pressão}

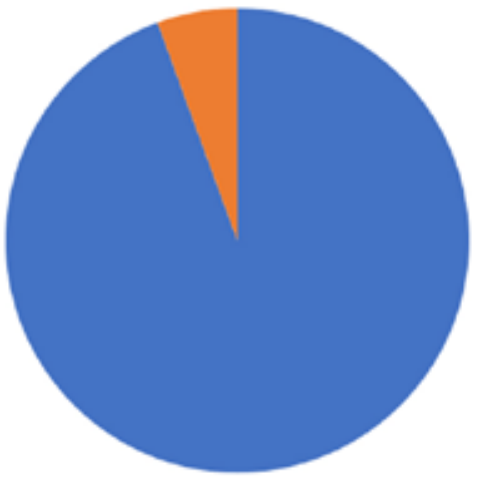

- Ausência de LPP 85 $(94,4 \%)$

= Presença de LPP 5 (5,6\%) 


\section{Gráfico 2. Incidência por sexo. Recife, PE, Brasil, 2018}

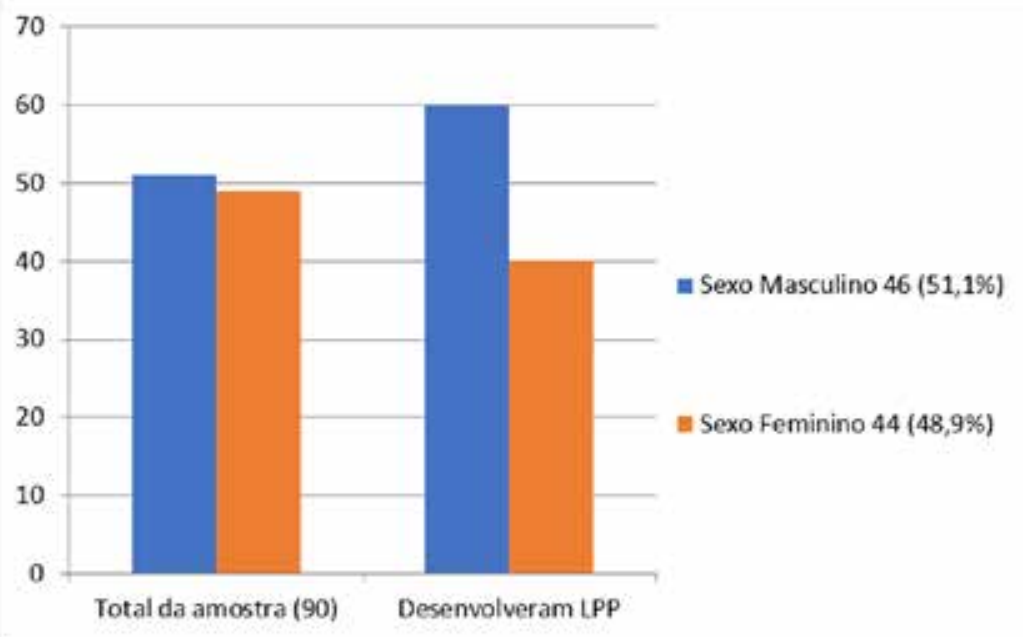

Gráfico 3. Incidência de LP por origem na unidade. Recife, PE, Brasil, 2018

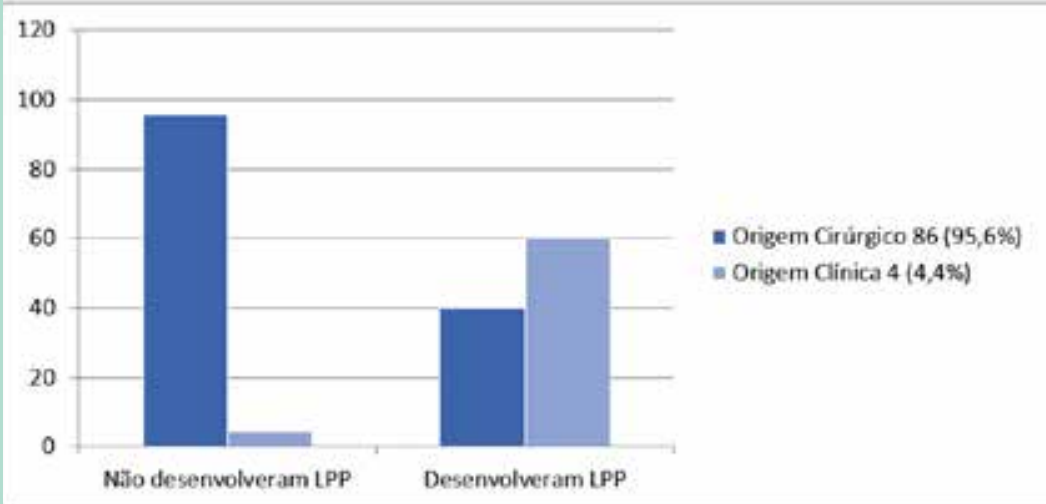

Gráfico 4. Incidência de LP por avaliação de risco. Recife, PE, Brasil, 2018

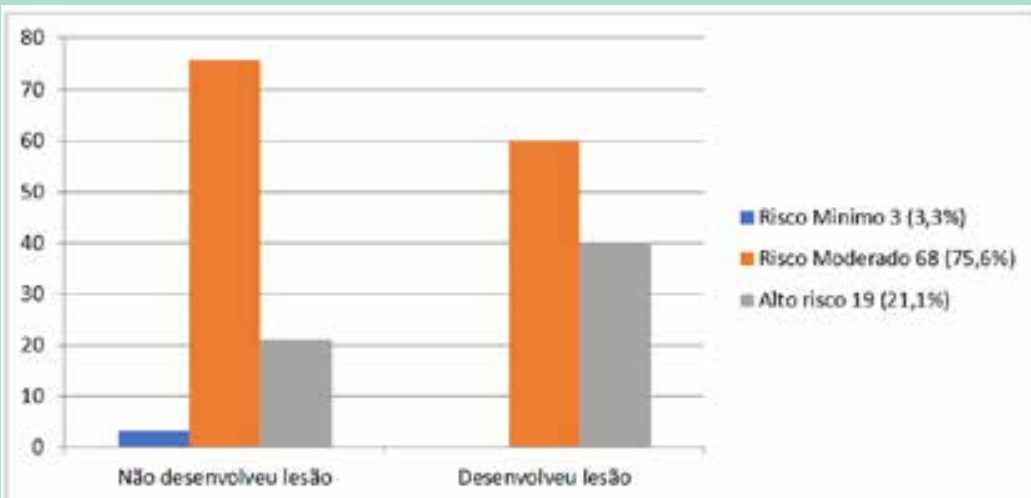

Foi observado que entre os pacientes da pesquisa, $46(51,1 \%)$ eram do sexo masculino e $44(48,9 \%)$ do feminino, referente aos que desenvolveram LP, $3(60 \%)$ eram do sexo masculino e $2(40 \%)$ do feminino, conforme Gráfico 2.

Acerca da origem destes pacientes, $86(95,6 \%)$ de origem cirúrgica e $4(4,4 \%)$ de origem clínica, destes, desenvolveram LP $3(60 \%)$ de origem clínica e $2(40 \%)$ de cirúrgica, conforme Gráfico 3.

Referente à escala de Braden, do total da amostra participante da pesquisa, $3(3,3 \%)$ apresentaram risco mínimo, $68(75,6 \%)$ risco moderado e $19(21,1 \%)$ apresentaram alto risco para o desenvolvimento de lesão, quando se refere aos que desenvolveram LP, $3(60 \%)$ risco moderado e 2 (40\%) alto risco. Conforme Gráfico 4.

De acordo com a avaliação do TISS, do total da amostra, $12(13,3 \%)$ apresentaram classe II, 18 (20\%) classe III, 3 classe IV (3,3\%), e 57 $(63,3 \%)$ sem avaliação, pois receberam alta antes das 24 horas, dos que desenvolveram LP, 4 (80\%) foram classificados com classe III e $1(20 \%)$ classe IV. Conforme Gráfico 5.

Acerca do motivo da saída deste paciente da UTI, do total da amostra, $82(91,1 \%)$ foram de transferência e 8 $(8,9 \%)$ de óbito, quando se refere aos que desenvolveram LP, 4 (80\%) foram a óbito e 1 (20\%) por transferência. Conforme Gráfico 6.

\section{DISCUSSÃO}

A presença de LP representa uma das principais complicações de pacientes internados em locais críticos, devido às suas condições clínicas graves ou necessidade de procedimentos mais rigorosos e frequentes, de caráter invasivo ou não.

Devido a estas lesões comprometerem o quadro clínico do paciente e aumentarem o tempo de internamen- 
Gráfico 5. Incidência de desenvolvimento de LP conforme gravidade. Recife, PE, Brasil, 2018

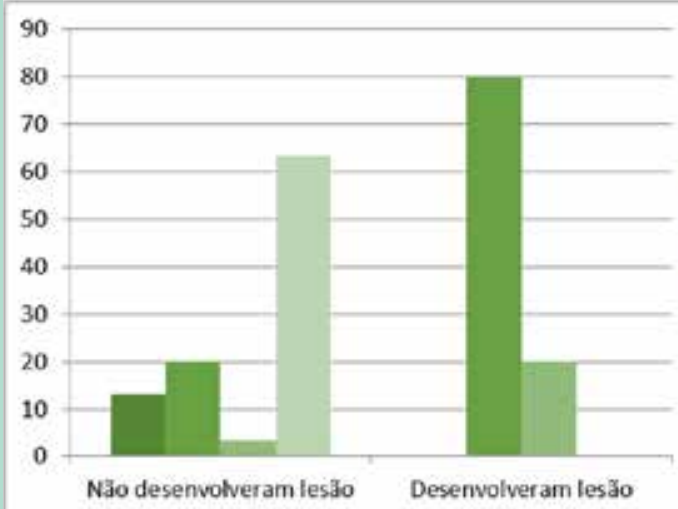

\section{a Classe II $12(13,3 \%)$}

= Classe ill 18 (20\%)

$=$ Classe IV $3(3,3 \%)$

Ne Nöo Classificado $51(63,3 \%)$

Desenvolveram lesăo

Gráfico 6. Incidência de saída de pacientes da unidade. Recife, PE, Brasil, 2018

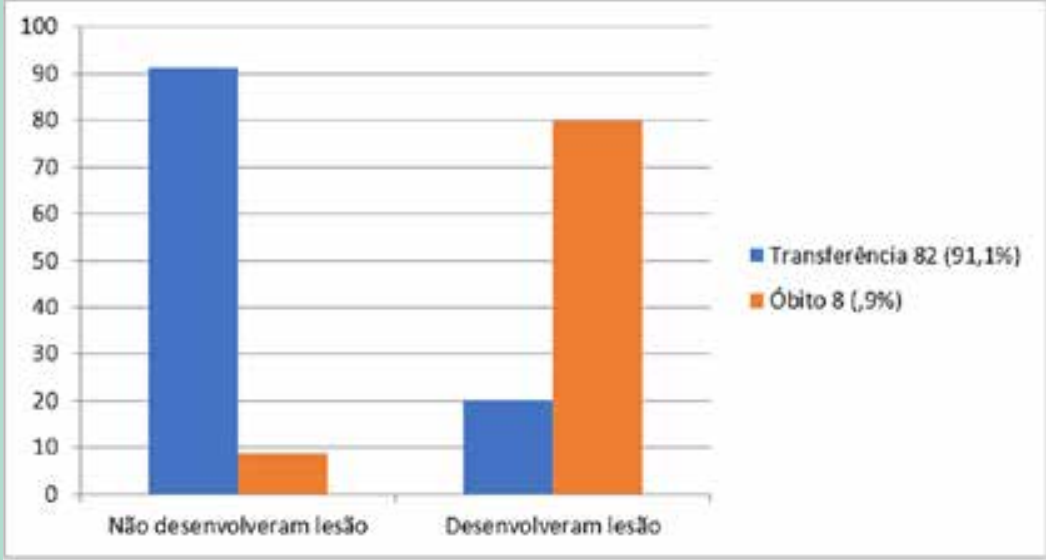

to, necessita-se de uma atenção direcionada da equipe multidisciplinar para a prevenção, aplicando sempre técnicas eficazes e estimulando a teoria do autocuidado.

Segundo dados da NPUAP, a prevalência de LPs em hospitais é de $15 \%$ dos pacientes admitidos, e a incidência é de $7 \%$. No Reino Unido, novos casos de LP acometem entre 4 a $10 \%$ das admissões. No Brasil, embora existam poucos trabalhos sobre incidência e prevalência, estudo realizado em hospital geral universitário evidenciou uma incidência de $39,81 \%$. Já em relação à incidência em UTI, estudos nacionais apontaterapia intensiva. para a prevenção e para que decisões sejam tomadas em caso de desenvolvimento de LP, além disto, a unidade hospitalar conta com uma equipe especializada de estomaterapia para acompanhamento destas lesões.

Nota-se que dos pacientes que desenvolveram LPs, a porcentagem maior foi de pacientes clínicos, verificando que os cuidados devem ser mais intensos e direcionados de forma cautelosa para com esta clientela, visto que a unidade na sua grande maioria recebe pacientes cirúrgicos.

As diretrizes internacionais e nacionais aconselham a utilização da escala de Braden para contribuir na identificação dos pacientes que apresentam o risco para LP desde admissão e durante o período de internação e aplicação das medidas preventivas pelos profissionais. Sugere-se que a reavaliação ocorra pelo menos a cada 48 horas após admissão ou sempre que as condições do paciente se modificar. Dado o exposto, foi visto que todos os pacientes apresentam algum risco para o desenvolvimento de lesão, e que dos que desenvolveram, nenhum paciente foi avaliado como risco mínimo, mas risco moderado e alto risco, o que também se precisa ter uma atenção sobre quais medidas estão sendo aplicadas quando os pacientes recebem esta avaliação.

Quanto ao critério de avaliação $62,5 \%{ }^{(13)}$. Face ao exposto, o resultado de 5,6\% identificado para incidência de LP na UTI investigada encontra-se abaixo do esperado para as unidades hospitalares e centros de

Essa baixa incidência pode ser atribuída a um conjunto de fatores e à importância do trabalho em equipe, além da Sistematização da Assistência da Enfermagem (SAE), que atua de forma contínua e promove uma recuperação positiva dos pacientes internados nesta unidade. A avaliação do enfermeiro realizada diariamente através da escala de Braden contribui pelo TISS, foi analisado que os pacientes que desenvolveram LP, receberam classificação III ou IV, significando pacientes que necessitam de cuidados intensivos, pacientes hemodinamicamente instáveis, necessitando um plano de ação diferenciado e direcionado, com participação da equipe multidisciplinar.

Dos participantes da pesquisa, foi visto que houve predomínio, como motivo da saída da UTI, a transferência, porém, dos que desenvolveram LP, o predomínio foi de óbito, significando que o quadro clínico do pa- 
ciente se encontrava grave, por isto, a importância da SAE, que atua de forma individualizada e direcionada para cada paciente, a fim de proporcionar um prognóstico positivo. Sabendo que existe relação direta entre o desenvolvimento da LP e a posição em que o paciente permanece por um maior período, pode-se concluir que os pacientes se mantiveram no leito por um tempo prolongado na mesma posição de decúbito, favorecendo o desenvolvimento da lesão. Tais fatos nos remetem à necessidade de avaliação e registro por parte da equipe acerca da condição hemodinâmica do paciente, bem como à importância de programação da mudança de decúbito conforme condição clínica, que deve ser avaliada a cada troca de plantão.

\section{CONCLUSÃO}

O estudo evidencia uma baixa incidência de LP em paciente internados na UTI, levando em consideração o referencial de estudos internacionais e nacionais. Observou-se também que o predomínio foi em pacientes clínicos e não cirúrgicos, que é a especialidade da UTI de estudo.

Embora exista uma atuação eficaz

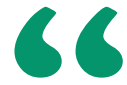

Tais medidas visam reduzir ainda mais a incidência de LP nesta unidade, principalmente em pacientes de origem clínica, reforçando a utilização do máximo de mecanismos para evitar o desenvolvimento destas lesões da equipe multidisciplinar, em especial da enfermagem que possui o instrumento da SAE e está com o paciente 24 horas por dia, identificando previamente os casos com risco para desenvolvimento da LP, ainda se fazem necessárias educação contínua e avaliação do protocolo estabelecido pela unidade para prevenção destas. Tais medidas visam reduzir ainda mais a incidência de LP nesta unidade, principalmente em pacientes de origem clínica, reforçando a utilização do máximo de mecanismos para evitar o desenvolvimento destas lesões, atuando de forma intensificada nestes pacientes que recebam um escore maior de acordo com o TISS e uma avaliação mais atenuada de acordo com a escala de Braden.

Percebe-se a importância de um trabalho em equipe, com planejamento estratégico, buscando sempre solucionar as falhas, não de forma isolada, mas em conjunto, seja na área médica, da enfermagem, nutricional, fisioterapia, e dos outros profissionais envolvimentos no cuidado do paciente internado nesta unidade. É essencial a atuação de forma holística, a fim de propiciar ao paciente uma boa recuperação e uma melhor qualidade de vida.

\section{Referências}

1. Ministério da Saúde, Secretaria de Vigilância Sanitária (BR). Portaria n. ${ }^{\circ} 332$ de 04 de Junho de 2000. Brasília (DF): MS, 2020.

2. Cook D, Rocker G. Dying with Dignity in the Intensive Care Unit. N Engl J Med. 2014; 370(26):2506-14.

3. Favarin SS, Camponogara S. Perfil dos Pacientes Internados na Unidade de Terapia Intensiva Adulto de Um Hospital Universitário. Rev Enferm UFSM. 2012; 2(2):320-9.

4. Araujo TG, Rieder MM, Kutchak FM, Filho JWF. Readmissões e óbitos após a alta da UTI - um desafio da terapia intensiva. Rev Bras Ter Intensiva. 2013; 25(1):32-8.

5. Moreira ET, Nunes TF, Santos ES, Calles ACN. Perfil E Gravidade Dos Pacientes Admitidos Em Unidades De Terapia Intensiva: Uma Revisão da Literatura. Cad Grad - Ciências Biológicas e da Saúde Fits. 2013; 1(2):45-52.

6. França EET, Ferrari F, Fernandes $P$, Cavalcanti $R$, Duarte $A$, Martinez $B P$, et al. Fisioterapia em pacientes críticos adultos: recomendações do Departamento de Fisioterapia da Associação de Medicina Intensiva Brasileira. Rev Bras Ter Intensiva. 2012; 24(1):6-22.
7. Neto M. Functional decline in intensive care unit (ICU). Rev Inspirar. 2013;5(23):1.

8. Ministério da Saúde, Agência Nacional de Vigilância Sanitária (BR). Segurança do Paciente - Úlcera por Pressão. Brasília (DF): MS/ANVISA, 2016.

9. Shahin ES, Dassen T, Halfens RJ. Incidence, prevention, and treatment of pressure ulcers in intensive care patients: a longitudinal study. Int J Nurs Stud. 2009; 46(4):413-21.

10. Costa IG. Incidência de úlcera por pressão em hospitais regionais de Mato Grosso, Brasil. Rev Gaúcha Enferm. 2010; 31(4):693-700.

11. Fernandes NCS, Torres C, Vieira D. Fatores de risco e condições predisponentes para úlcera de pressão em pacientes de terapia intensiva. Rev. Eletr. Enf. 2008; 10(3):733-46.

12. Luz SR, Lopacinski AC, Fraga R, Urban CA. Úlceras de pressão. Geriatria \& Gerontologia. 2010; 37-42.

13. Matos LS, Duarte NLV, Minetto RC. Incidência e prevalência de úlcera por pressão no CTI de um Hospital Público do DF. Rev. Eletr. Enf. 2010 out/dez;12(4):719-26. 